\title{
SOBRE EL DESARROLLO SOSTENIBLE Y LA SOSTENIBILIDAD: CONCEPTUALIZACIÓN Y CRÍTICA
}

\author{
ON SUSTAINABLE DEVELOPMENT AND SUSTAINABILITY: \\ CONCEPTUALIZATION AND REVIEW
}

\author{
Iván López Pardo \\ Universidad de Zaragoza. España/Spain \\ ivalopez@unizar.es
}

Recibido/Received: 01/06/2015

Modificado/Modified: 22/09/2015

Aceptado/Accepted: 04/10/2015

\section{RESUMEN}

Hace algo más de tres décadas, y con especial intensidad en los años 80 , se inicia un proceso sin precedentes de reflexión a nivel mundial que impulsa Naciones Unidas, seguida de la Unión Europea, para abordar la cuestión de la sostenibilidad y el desarrollo sostenible del planeta como uno de los grandes retos a los que se enfrentan las sociedades del siglo XXI. La publicación del Informe Brundtland (WCED, 1987) representa un hito en dicho proceso al ofrecer una definición del desarrollo sostenible que hasta la actualidad es referencia en el debate. En este artículo se recogen las posiciones y argumentos de defensores y críticos para arrojar luz sobre un concepto en continuo estado de definición a la vez que plantear los interrogantes por resolver en un debate ni mucho menos acabado.

\section{PALABRAS CLAVE}

Sostenibilidad, desarrollo sostenible, Informe Brundtland, protección ambiental, equidad.

\section{SUMARIO}

1. Introducción. 2. El concepto de sostenibilidad y desarrollo sostenible en el Informe Brundtland: orígenes y dificultades. 2.1. La capacidad de transformación del concepto de sostenibilidad y desarrollo sostenible. 3. La cuestión sin resolver de las contradicciones entre crecimiento económico y protección ambiental en el Informe Brundtland. 4. Las necesidades de las generaciones futuras: la equidad inter e intrageneracional. 4.1. La cuestión de la equidad y el desarrollo sostenible. 5. Conclusiones. Bibliografia.

\begin{abstract}
For more than three decades, and with particular intensity in the 80 's, starts a process with no precedent on the global reflection driven by United Nations, and followed by the European Union, to address the issue of the planet's sustainability and sustainable development as one of the great challenges that the XXI century face. The publication of the Brundtland Report (WCED, 1987) represents a milestone in the process offering a definition of sustainable development until today reference in the debate. In this article we review the positions and arguments for and those that are critical of this definition, to shed light on a concept in continuous state of definition at the same time pose the questions to be resolved in a debate no at all concluded.
\end{abstract}

\section{KEYWORDS}

Sustainability, sustainable development, Brundtland Report, environmental protection, equity. 


\section{CONTENTS}

1. Introduction. 2. The concept of sustainability and sustainable development in the Brundtland Report: origins and difficulties. 2.1. The transformation capacity of the sustainability and sustainable development notion. 3. The unresolved issue of the contradictions between economic growth and environmental protection in the Brundtland Report. 4. The needs of future generations: inter- and intragenerational equity. 4.1. The issues of equity and sustainable development. 5. Conclusions. References.

\section{INTRODUCCIÓN}

Hace algo más de tres décadas, y con especial intensidad en los años 80 , se inicia un proceso sin precedentes de reflexión a nivel mundial que impulsa Naciones Unidas, seguida de la Unión Europea, para abordar la cuestión de la sostenibilidad y el desarrollo sostenible del planeta como uno de los grandes retos a los que se enfrentan las sociedades del siglo XXI.

El debate es motivado por la progresiva toma de conciencia en torno a las limitaciones en "el estado de la tecnología y la organización social, o la capacidad del medio ambiente para satisfacer las necesidades actuales y futuras" (WCED, 1987: 43), y las incompatibilidades entre crecimiento económico y protección medioambiental y las posibilidades de mantener a medio o largo plazo el grado de crecimiento actual de los países con economías más desarrolladas (Faucheux et al., 1998: 6; Ekins et al., 1998: 17) son realidades.

La publicación del Informe Brundtland (WCED, 1987) - también llamado Nuestro Futuro Común (Our Common Future) representa un hito en dicho proceso. En el mismo, la Comisión Mundial sobre el Medio Ambiente y el Desarrollo de la ONU define el desarrollo sostenible como "...el desarrollo que satisface las necesidades de la generación actual sin comprometer la capacidad de las generaciones futuras para satisfacer sus propias necesidades." (WCED, 1987: 8).

Se trata de la definición más comúnmente utilizada en la esfera política, institucional y científica tanto nacional como internacional en el intenso y rico debate sin precedentes sobre la sostenibilidad planetaria (Lamberton, 2005; Lafferty et al., 1999) que junto con el Banco Mundial en el documento Environmental Growth and Development (Banco Mundial, 1987) y posteriormente en la Cumbre de la Tierra en 1992, darán forma a la necesidad de una nueva visión del mundo que sirva como base global para el consenso, y que eventualmente nos aproximará al término "desarrollo sostenible" (Mebratu, 1998: 503).

En el presente artículo abordamos la noción de desarrollo sostenible y sostenibilidad - que a efectos de facilidad expositiva, se va aquí a considerar como equivalentes los términos 'desarrollo sostenible' y 'sostenibilidad' o 'sustentabilidad', si bien, se trata de conceptos ligeramente distintos como más adelante en el documento se explica - a partir de sus dimensiones o aspectos que más atención ha recibido en los últimos años por parte de la comunidad científica e instituciones. Tratamos así de presentar una visión crítica sobre el tema que puede arrojar luz sobre lo conseguido hasta el momento así como las carencias en su recorrido epistemológico.

Presentamos los argumentos tanto de sus defensores y los críticos o detractores de este concepto para concluir que la principal aportación de la noción de sostenibilidad y desarrollo sostenible del Informe Brundtland es haber mantenido vigente durante todos estos años y hasta la actualidad el debate y discusión pública en torno a la necesidad de su inclusión en la agenda política y social a escala tanto local como nacional o internacional. 


\section{EL CONCEPTO DE SOSTENIBILIDAD Y DESARROLLO SOSTENIBLE EN EL INFORME BRUNDTLAND: ORÍGENES Y DIFICULTADES}

Definir el desarrollo sostenible se presenta como una tarea difícil (Kane, 1999), quedando de manifiesto 1) la pugna semántica en torno al concepto y su complejidad (Lafferty et al., 1999); 2) el amplio "abanico" de posturas y propuestas tanto teóricas como empíricas, o las múltiples definiciones que coexisten en la literatura tanto científica como institucional (Giddings et al., 2002; Hopwood et al., 2005); 3) y ello quizá debido no solo de la polisemia del principio, sino de su misma amplitud y alcance (Arias Maldonado, 2004: 123).

Si bien en la literatura ha prevalecido la atención dirigida ante todo a la definición "corta" de sostenibilidad y el desarrollo sostenible del Informe Brundtland, la interpretación y análisis de esta noción cabe aproximarlo desde una visión "amplia" que contemple el conjunto de este Informe y las múltiples definiciones del desarrollo sostenible que se recogen en el mismo (Lafferty et al., 1999).

Una de las consecuencias de lo anterior es la evidencia del desacuerdo profesional, en particular a la hora de poner la idea del desarrollo sostenible en operatividad (Banuri, 1999: 1), al igual que el predominio del desarrollo económico - o de la cuestión de cómo mantener el ritmo de crecimiento actual de las naciones más desarrolladas económicamente - en debate el debate sobre la sostenibilidad (Edwards-Jones et al., 2000).

Previo a su popularización, las premisas que conforman la noción de sostenibilidad cabe reconocerlas en el concepto de ecodesarrollo propuesto por Maurice Strong en el marco del Programa de las Naciones Unidas para el Medio Ambiente (PNUMA, 1973). A lo largo de los años 70 y en el marco del United Nations Environment Programme (UNEP), el ecodesarrollo pone el foco de atención en la importancia de los objetivos sociales del desarrollo sostenible y así también de la distribución de la renta, al igual que en las limitaciones ecológicas y de eficiencia económica que caracterizan al modelo contemporáneo de crecimiento (Sach, 1991, 1994: 26-28; Centre de Cooperation internationale en Recherche Agronomique pour le Developpement, 1986; O'Connor et al., 1998: 42:52; Lafferty et al., 1999: 214).

Con ello, la noción de ecodesarrollo posibilita una aproximación crítica y amplia del desarrollo tradicionalmente acotado por ejemplo al Producto Interior Bruto, la explotación sin límites de los recursos naturales o la modernización tecnológica (O'Connor et al., 1998: 41).

Bajo el giro argumentarlo y motivada por la Conferencia Internacional sobre la Población y el Desarrollo celebrada por Naciones Unidas en Bucarest (CIPD -1974), la Declaración de Cocoyot es el primer intento institucional por acotar y precisar el concepto de sostenibilidad y desarrollo sostenible.

No obstante, no será hasta que forme parte de la Estrategia de Conservación del Mundo (1980) cuando la noción de desarrollo sostenible juegue el papel central que en la actualidad sigue ocupando en la política internacional y quede vinculado a la idea por la cual "para que el desarrollo sea sostenible, debe de tener en cuenta los factores sociales y ecológicos, así como los económicos, en base a los recursos naturales vivos y los no vivos, así como las ventajas y desventajas a largo plazo, al igual que a corto plazo, de la acción [humana] alternativa" (IUCN, 1980: 4).

Se trata esta de una idea que guiará en años posteriores aproximaciones de referencia al concepto como las que destaca Mebratu (1998) y distingue atendiendo a su visión institucional -ejemplifica por la World Commission on Environment and Development 
(WCED), el International Institute of Environment and Development (IIED), el World Business Council for Sustainable Development (WBCSD) y la Unión Internacional para la Conservación de la Naturaleza (UICN, 1991)-, la visión ideológica -que distingue entre ideología "verde" y las versiones ambientales de las ideologías clásicas de emancipación como (como por ejemplo la teología de la liberación, el feminismo radical, o el marxismo)- o la visión académica -que reflejan la respuesta de la comunidad científica al reto de la crisis ambiental en el siglo XXI (ver Tabla 1).

\begin{tabular}{lllll}
\multicolumn{2}{l}{ Tabla 1: Análisis comparativo de la visión institucional de la Sostenibilidad } \\
\hline Institución Conductores & $\begin{array}{l}\text { Solución } \\
\text { Epicentro }\end{array}$ & $\begin{array}{l}\text { Solución } \\
\text { Plataforma }\end{array}$ & $\begin{array}{l}\text { Instrumentos } \\
\text { (Liderazgo) }\end{array}$ \\
\hline WCED & $\begin{array}{l}\text { Consensos } \\
\text { políticos }\end{array}$ & $\begin{array}{l}\text { Crecimiento } \\
\text { sostenible }\end{array}$ & Estado-nación & $\begin{array}{l}\text { Organizaciones } \\
\text { internacionales y gobiernos }\end{array}$ \\
IIED & $\begin{array}{l}\text { Desarrollo } \\
\text { rural }\end{array}$ & $\begin{array}{l}\text { Atención } \\
\text { medioambiental } \\
\text { primaria (PEC) }\end{array}$ & Comunidades & $\begin{array}{l}\text { ONGs nacionales e } \\
\text { internacionales }\end{array}$ \\
WBCSD & $\begin{array}{l}\text { Interés } \\
\text { comercial }\end{array}$ & Eco-eficencia & $\begin{array}{l}\text { Comercio e } \\
\text { industria }\end{array}$ & Liderazgo corporativo \\
\hline
\end{tabular}

Fuente: Mebratu, 1998

\subsection{La capacidad de transformación del concepto de sostenibilidad y desarrollo sostenible}

En el marco de la reflexión en torno a la noción de sostenibilidad y desarrollo sostenible, buena parte del diverso abanico de argumentos resultantes se vertebran en torno a la cuestión de la capacidad del concepto para la transformación o el cambio social. En particular, lo hace a partir de dos componentes o espacios discursivos como son, por una parte, el del significado que cabe atribuir al término desarrollo sostenible, y por otra parte el de las condiciones necesarias para la sostenibilidad (Mitlin, 1992). A éstas se añade la necesidad de una norma sobre una ética global (Lafferty et al., 1999).

Como a continuación se comprueba, la capacidad del término para conciliar posturas, o por el contrario de generar disenso queda a su vez bajo debate. En este caso, nos referimos a la dualidad entre la integración y la fragmentación que ha caracterizado la reflexión durante todos estos años (Rosenau, 2003) -dicotomía que refleja los elementos persistentes que identifican a la sostenibilidad y que facilitan su estudio epistemológico.

En primer lugar, desde las posiciones más críticas, se cuestiona la intención 'real' del concepto de sostenibilidad y desarrollo sostenible para cambiar las tendencias insostenibles y contradicciones intrínsecas a la interacción entre desarrollo económico y deterioro medioambiental, o la cultura y estilos de vida que las sustentan de las sociedades contemporáneas (Mitcham, 1995: 322; Foladori, 1999: 19; Hopwood et al., 2005: 40; Sneddon et al., 2006: 260; O'Connor, 2002: 28; Burgess et al., 1998).

El argumento central al que se apela es el exceso de normatividad del concepto, su ambigüedad, vaguedad, falta de precisión o de nuevo las contradicciones que conlleva el término (O'Connor, 2002; Gow, 1992; Mozaffar, 2001; Daly, 1996; Dresner, 2002; Naredo, 1996; Goldin et al., 1995; Tryzna, 1995; Redclift et al., 1997; Redclift, 1987).

No se puede decir hay un entendimiento común sobre la esencia del desarrollo sostenible, o los medios para alcanzarlo, pero tampoco sobre la importancia del desarrollo sostenible como meta social y comparada con las otras metas sociales - la forma y fondo que adopta la transformación social que requiere como objetivo, o la profundidad y alcance de los cambios 
en el modelo económico y de desarrollo actual (Davidson, 2000; Harrison, 2000; Bebbington, 2001; Carvalho, 2001; Giddings et al., 2002); son persistentes las diferencia entre grupos, comunidades, países y sistemas internacionales a la hora de implementar los compromisos adquiridos y la controversia en que de todo ello resulta (Sachs, 2002); y quedan un "incuestionable conjunto de valores" por definir, confundiéndose en el debate si es la economía lo que se quiere sostener o la naturaleza (Rosenau, 2003: 16).

Siempre desde esta perspectiva crítica, las expectativas creadas y el posterior desencanto y frustración en torno al concepto -con políticas que no han supuesto cambios reales sobre sobre los principales objetivos establecidos en la Cumbre de la Tierra en 1992, la falta de capacidad y compromiso de los gobiernos y organizaciones internacionales, o la burocratización y alta influencia de los grupos económicos y políticos de interés de interés en los procesos de decisión- han contribuido a su actual estado ambiguo y controvertido (Godard, 1998: 299; Lafferty et al., 1999: 215; Berkhout et al., 2003; Scoones et al., 2007; Jordan et al., 2009; Volger y Jordan, 2003; Redclift, 1987, 1992; Kenny et al., 1999).

Por otra parte, bajo esta línea argumentativa, es patente la utilización en exceso del término, como etiqueta o cliché, haciendo que se desvirtúe y pierda valor (Lélé, 1991; Luke, 1995; Sneddon, 2000; Fernando, 2003; Holmberg et al., 1994; Mitcham, 1995; O'Connor, 2002).

Nos encontraríamos por tanto ante una "falsa noción" del desarrollo sostenible, un concepto "tan ambiguo como el capitalismo sostenible y sus conceptos hermanos como los de 'agricultura sostenible', 'energía sostenible y utilización de recurso', y 'desarrollo sostenible", de manera que "el término puede ser utilizado para cualquier cosa que uno quiera que signifique, lo cual es parte de su atractivo" (O'Connor, 2002: 27).

Igualmente, la alteración de la noción de la sostenibilidad quedaría reflejada en el hecho de resultar "el desarrollo sostenible en un concepto vergonzosamente antropocéntrico" que se ocupa de definir en otros ámbitos las necesidades desde el punto de los humanos (Lélé, 2000: 32), o por su análisis y estudio ante todo desde una perspectiva cuantitativista (Sneddon et al., 2006)

Ante la complejidad de retos como el de la sostenibilidad planetaria y el desacuerdo sobre lo que debe de ser sostenido, la operatividad -y por tanto la capacidad de transformación social del concepto- queda mermada por las dificultades que implica para medir la sostenibilidad o traducir la meta de la sostenibilidad en pasos o fases concretas para su consecución (Villanueva, 1997; Campbell, 1996; Redclift, 1993; Sachs, 1999; Satterthwaite, 1996).

Una vez más, los críticos mantienen que nos encontramos ante una "ilusión política" que fracasa en afrontar las contradicciones básicas de cómo reconciliar la sociedad industrial con las limitaciones de los sistemas ecológicos del planeta (Richardson, 1997: 57; Sneddon et al., 2006: 260; O’Riordan, 1993: 140). Más aún, la sostenibilidad y el desarrollo sostenible han sido una buena estrategia política para dar continuidad al modelo de desarrollo existente o justificar y legitimar políticas y prácticas que van desde la utopía agraria comunal hasta el desarrollo del mercado a gran escala de tipo capitalista intensivo (Daly, 1996; Hopwood et al., 2005:40; Worster, 1995; Sivaraksa, 1992; Baeten, 2000; Jabareen, 2008; Redclift, 1993:7).

En consecuencia, resulta difícil clasificar al desarrollo sostenible como fuerza globalizadora, dado que la globalización tiene lugar desde dos sinergias básicas al mismo tiempo que contrarias: una tendente a la centralización, integración y globalización, y otra 
hacia la descentralización, fragmentación y localización, polaridades que pueden verse bien como premisas políticas o como procesos empíricos (Rosenau, 2003: 14).

De otra parte, los defensores del concepto ponen la atención en la generalización del consenso alcanzado a nivel global en torno a al desarrollo sostenible como objetivo deseable -como meta común a alcanzar- la cualidad integradora del concepto resulta esencial por tratarse de un ideal o concepto político persistente con capacidad para conseguir situar a la cuestión de la sostenibilidad planetaria como una de las prioridades de la agenda política internacional -las preocupaciones de la sostenibilidad han sido puestas en el centro de una comprensión normativa del cambio social y político, en el marco de la relación entre medio ambiente, bienestar y las luchas por la justicia social-, o al menos objeto de debate y reflexión de trascendencia para la opinión pública, haciendo del mismo el elemento central en la redacción de políticas tanto nacionales como internacionale -en lo que denominan como un "juego global" y una "nueva política"- con mayor potencial antes incluso que en el ámbito científico (O’Riordan, 1993; Mebratu, 1998: 494; Gibbs, 1998: 135; CEC, 1992; Stow, 1989; Dobson, 1999; Lafferty et al., 1999; Howarth y Wilson, 2006; Sneddon et al., 2006: 260; Newman, 2006: 634).

Es en la fuerza ética del concepto donde recae su legitimidad y potencial para superar el conformismo con las normas a través del compromiso con la política democrática (Wetlesen, 1993; Cohen, 1971: 180); en su papel para la consolidación de un "nuevo pensamiento" que incide en todos los aspectos de la vida social y reta la creciente integración del mundo en torno a la economía capitalista (Myerson et al., 1996: 103; Middleton et al., 1993; Christie et al., 2001).

En consecuencia, es posible afirmar la instrumentalidad del concepto al reconocer la formación de una "visión global" que proporciona del futuro del planeta (Dobson, 1999) a través de la sostenibilidad como noción abstracta -que todavía requiere de elaboración y definición- y universalmente deseada (Lafferty, 2004; Sneddon et al., 2006; Jabareen, 2008).

A tenor de estos argumentos, la flexibilidad, vaguedad o la falta de precisión a la que aluden los críticos representa una virtud, más si cabe al haberse demostrado a lo largo de estos años que posibilita el trabajo sobre una base común para la discusión de una amplia variedad de actores sociales -normalmente con posturas e intereses contrapuestos- tanto en el ámbito de desarrollo económico como del ambientalismo (Pezzoli, 1997).

Así, desde que la sostenibilidad se definiera en el Informe Brundtland, "el valor de preservar el medio ambiente y mantener su viabilidad es ampliamente compartido en todos los niveles de la comunidad [internacional]" (Rosenau, 2003: 16).

Es lo que Dale (2001) ha denominado la ambigüedad constructiva, recordando el dilatado consenso en torno a la comprensión del concepto y el hecho de clamar por una ética global de la sostenibilidad como prerrequisito para el cambio, impulsando la adopción que apoya de una "asociación mundial" (global partnership) para el desarrollo sostenible promulgado en la Cumbre de Río (Wetlesen, 1993).

Como resultado, bajo el corpus de argumentos favorables al concepto de sostenibilidad y desarrollo sostenible, la capacidad de transformación y cambio social de esta noción se traduce ante todo en su influencia sobre la agenda política que se configura a partir del nuevo discurso de los derechos en torno al desarrollo, integra la conciencia sobre la sostenibilidad prestando atención a los derechos y condiciones socioeconómicas y ambientales del ámbito local sobre el global, con una visión a largo y de responsabilidad sobre las generaciones futuras, e incorporando en la reflexión el rendimiento de cuentas (accountability) (Kabeer, 2005); que aboga por el diálogo interdisciplinar e intercultural, y así involucrando los ámbitos académicos, pero a su vez político, económico y social y que 
recoge tanto a las ciencias sociales como a las naturales, la legislación, la política o la gestión (Cendra Garreta et al., 2006: 2; Dalal-Clayton, 2004).

Un ejemplo de ello son las acepciones más 'fuertes' o más 'débiles', la más coherentes o menos congruentes con los principios por los que aboga la sostenibilidad como meta a alcanzar.

\section{LA CUESTIÓN SIN RESOLVER DE LAS CONTRADICCIONES ENTRE CRECIMIENTO ECONÓMICO Y PROTECCIÓN AMBIENTAL EN EL INFORME BRUNDTLAND}

Del conjunto de argumentos antes expuestos cabe detenerse en la reflexión en torno al concepto de sostenibilidad y desarrollo sostenible del Informe Brundtland que se desenvuelve con especial intensidad atendiendo a la cuestión de su capacidad para abordar las contradicciones propias de la interacción entre desarrollo económico y protección medioambiental -debate que se amplía a la esfera de 'lo social' o las condiciones de vida de la población.

A la hora de analizar los dilemas propios a la interrelación entre ambos espacios, es necesario distinguir dos planos como son el conceptual o semántico, y el del mercado o de su traducción empírica. En el primero de ellos -el campo semántico- quedan en evidencia las contradicciones propias de la intersección entre los conceptos de 'desarrollo' y 'sostenible', o la posibilidad de simultaneidad entre desarrollo económico y conservación ecológica (Lélé, 1991; Lafferty y Meadowcroft, 2000; García et. al, 2004).

En concreto, en el Informe, la ambigüedad del concepto de desarrollo sostenible ejemplifica la difícil conciliación entre ambos espacios por dos razones: 1) la existencia de límites sobre los estilos de vida incompatibles con la conservación de la naturaleza; 2) el apoyo que se lleva a cabo del crecimiento económico para la satisfacción de las necesidades humanas, término este, el de necesidades, que, como se ha visto, queda sin precisar (Luffiego García et. al, 2000: 474).

No obstante, a su vez tres premisas que hacen que el concepto cuestione el modelo convencional de desarrollo: 1) al integrar los conceptos de desarrollo, bienestar social y calidad de vida; 2) al reclamar el reparto o distribución equitativa de la riqueza, tanto sobre las generaciones presentes como sobre las futuras; y 3) y por poner como condición la racionalización en la utilización de los recursos como condición para contar con un Planeta habitable a largo plazo (Font et al., 2000).

El desarrollo sostenible así presentado supone un principio sólido para la acción colectiva y la resolución de los conflictos o crisis entre el medio ambiente y el desarrollo y la visión de que sería deseable un mundo justo y un mundo ambientalmente sostenible (Sneddon et al., 2006: 260-261).

Antes de continuar con esta línea argumentativa, cabe detenerse por un momento en la comprensión más precisa del desarrollo y el crecimiento. Riggs (1984), a partir de la revisión de la literatura económica, asegura que el término desarrollo reemplazó ampliamente a términos anteriores como los de 'progreso' o 'evolución'. Más aún, apunta que términos como los de 'cambio', 'crecimiento', 'transformación', 'occidentalización', ‘industrialización’ y ‘modernización', están estrechamente ligados a la idea de ‘desarrollo’ y con connotaciones similares si no idénticas. 
Por su parte, Ekins et al. (1998a: 17) distinguen tres tipos de crecimiento, como son el de la economía biofísica, el de la producción o la renta (PIB), y el del bienestar humano, los tres en continuo estado de interacción.

La síntesis es bien formulada por O'Connor (1994), en primera instancia formulando dos premisas. Por una parte, que los procesos históricos de industrialización (o de desarrollo económico) suelen ser contrarios o antagónicos a prestar atención a su interacción con la ecología, ya sea por el volumen de materiales que requieren dichos procesos, o bien por sus efectos a través de los desechos, y queda en cuestión la posibilidad de la preservación del capital ecológico existente, al menos en el horizonte del largo plazo.

Por otra parte, las economías humanas dependen del capital natural para su funcionamiento y crecimiento, de los recursos naturales y de su utilización, al mismo tiempo que la mayoría de los ciclos de la biosfera dependen o están en estrecha dependencia de los procesos industriales o de la agricultura humana.

Este mismo autor (O'Connor,2002: 28) reconoce al menos cuatro sentidos del término 'sostener', que puede referirse a 1) mantener los procesos de acumulación de capital en el contexto de la globalización, y ello en unas pocas manos; 2) facilitar recursos a poblaciones del planeta que los necesiten y mantenerlas; 3) o a preservar la explotación laboral, económica y mercantil de segmentos amplios de la sociedad; 4) y con una cuarta acepción relativa a la 'sostenibilidad ecológica'.

A su vez, en el plano de la traducción empírica, Victor (1991) recuerda que en términos de la economía clásica, la renta o el bienestar sólo pueden ser mantenidos a largo plazo si el stock de capital del que se parte no declina. Ahora bien, ¿de qué stock de capital se está hablando? No solo el capital hecho por el ser humano, sino que igualmente bienes y capacidades del medio ambiente natural, el capital natural que, en definitiva, no ha sido producido por los humanos en primera instancia, ni reproducido.

El análisis del concepto de desarrollo sostenible, en especial desde indicadores como el Producto Interior Bruto (PIB), plantea al menos dos interrogantes: si necesariamente un mayor PIB implica a su vez más bienestar y mayor protección medioambiental; o de manera inversa, si la sostenibilidad medioambiental incrementa o reduce el bienestar de los países occidentales; o si las condiciones de compatibilidad de ambos objetivos serían menos restrictivas que las de la sostenibilidad y el PIB (Jacobs, 1991; Ekins, 1993).

Una idea muy extendida es que los países en desarrollo deben anteponer el crecimiento económico a las preocupaciones medioambientales, dado que cuanto mayor su nivel de renta, mejor trato harán de los conflictos medioambientales. Se trata esta de una premisa difícil de defender, cuando se comprueba, por ejemplo, que Estados Unidos, el país con ratios muy elevados de renta, cuenta con promedios incluso superiores de contaminación.

En lo que Jabareen (2008) denomina como la 'paradoja ética del concepto', no queda clara la compatibilidad de la unión de los dos términos bajo un solo concepto. El esfuerzo se dirige a reconciliar dos espacios que históricamente han tenido una relación antagónica, como son los de medio ambiente y desarrollo, y donde si bien es manifiesto el origen y significado ecológico del término sostenibilidad, no lo es el de desarrollo, que en especial resulta impreciso desde su dimensión no solo semántica, sino también política y moral (Figura 1).

Una distinción que arroja luz en lo anteriormente expuesto es la dicotomía entre antropocentrismo y ecocentrismo. La primera orientación - la antropocéntrica - queda ejemplificada por la Estrategia Mundial para el Desarrollo (IUCN) que define el desarrollo como "la modificación de la biosfera y la aplicación de los recursos humanos, financieros 
y recursos no vivos para satisfacer las necesidades humanas y mejorar la calidad de la vida humana" (IUCN, 1980: sección 1, párrafo 3).

Figura 1: Marco conceptual del Desarrollo Sostenible

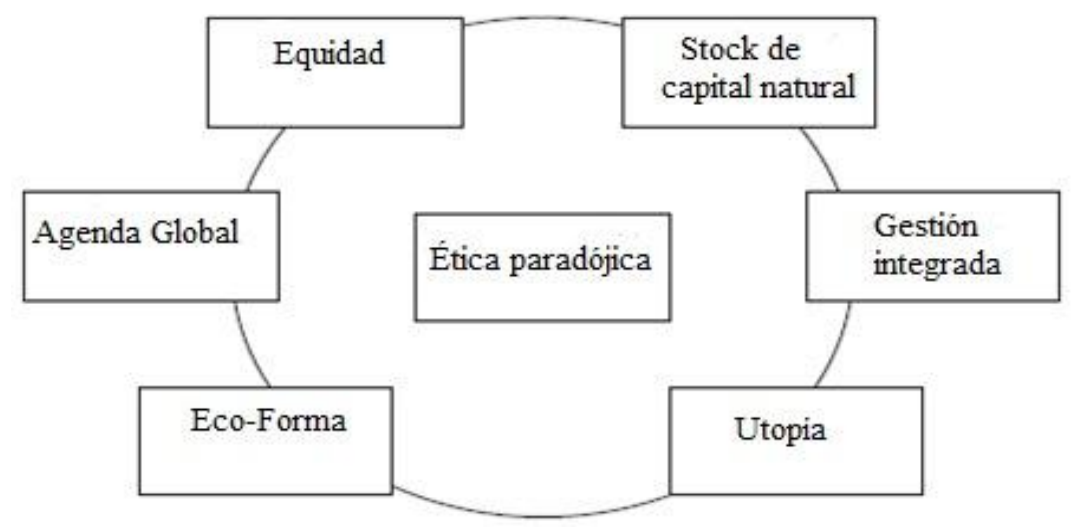

Fuente: Jabareen, 2008

Si bien se trata de una definición mediante la cual lo que en primer lugar importa es mantener un nivel determinado de bienestar humano intra- e intergeneracional, cabe matizar que en particular esta Estrategia lo hace desde la necesidad a la que apela de su compatibilidad con el equilibrio ecológico; más aún, recoge que "el desarrollo y la conservación operan en el mismo contexto global, y los problemas subyacentes que deben ser superados [deben entenderse como] idénticos si se quiere tener éxito" (IUCN, 1980: sección 20, párrafo 1), lo que permite afirmar que conlleva un carácter integrador los objetivos sociales y ecológicos.

A su vez, en cuanto a la acepción ecocéntrica, Hueting (1986) defiende la posibilidad de mantener el bienestar mediante la sostenibilidad medioambiental, incluso haciendo caer la producción económica, y así pone de relieve la dependencia de la producción, el medio ambiente y la seguridad del futuro de la sostenibilidad medioambiental.

Ambas visiones -la versión antropocéntrica y la ecocéntrica- implican versiones 'débiles' de la sostenibilidad, dado que en ninguno de los dos casos aspiran a resolver los conflictos inherentes a la interacción entre preservación del entorno natural y desarrollo económico (Dobson, 1998); o tampoco inciden en la distribución equitativa de la riqueza entre regiones del planeta como solución o parte de la solución al problema al conflicto, o sobre el dilema protección medioambiental frente a justicia social (Paehlke, 1995: 135).

La solución al dilema según Lafferty et al. (1999: 3) es que "la sostenibilidad es condición o restricción del desarrollo humano, e implica un sentido positivo del desarrollo". Desde el marco más amplio de análisis, O'Connor et al. (1998: 34) estiman que el término de desarrollo sostenible puede no obstante contemplar cuestiones como la de la justicia en la distribución ecológica, o la sostenibilidad económica, ecológica y social en el tiempo, las asimetrías en la distribución de los recursos, o los procesos de mercantilización y capitalización de la naturaleza como respuesta a las sociedades de mercado en crisis ambiental.

A este respecto, las ciencias ecológicas subrayan dos espacios críticos. De una parte, las tendencias sociales hacia el individualismo, economicismo y optimismo tecnológico 
(Frazier, 1997; Faber et al., 2002; Dawe et al., 2003, Robinson, 2004). De otra parte, la agenda genérica y tradicional de los ambientalistas más activos a la hora de diseñar las "políticas verdes", más precisamente en proyectos como la Agenda 21 Local, resultan insuficientes para abordar los retos ambientales a nivel global (Selman, 2000: 40; Mebratu, 1996, 1998; Lafferty et al., 1999).

En este sentido, la capacidad de cambio y transformación del concepto de sostenibilidad y desarrollo sostenible queda limitada por la aplicación del concepto mediante estrategias ante todo de sostenibilidad local y así desplazando la mayor responsabilidad a las autoridades locales (Van der Straaten, 1989: 69; Wallner et al., 1996).

A las perspectivas antropocéntricas y ecocéntricas, cabe añadir la democéntrica. Si bien no es objeto de este artículo explicarlas en detalle, a modo de ejemplo cabe mencionar a Arias Maldonado (2004) que propondrá la dicotomía entre la sostenibilidad normativa y la tecnocrática o cerrada; entre la definición de sostenibilidad resultado de la deliberación pública y aquella producto de la ciencia o ideología; o una relación con la democracia 'fuerte' o necesaria, o bien 'débil' y contingente; o finalmente por su orientación dominante bien procesual o bien finalista (ver Tabla 2).

Tabla 2: Modelos de Sostenibilidad y su relación con la democracia

\begin{tabular}{lll}
\hline & \multicolumn{1}{c}{$A$} & \multicolumn{1}{c}{$B$} \\
\hline Tipo de sustentabilidad........................ & Normativa/abierta & Tecnocrática/cerrada \\
Determinación del contenido............... & Deliberación pública & Ciencia/Ideología \\
Relación con la democracia ................... & Fuerte/necesaria & Débil/contingente \\
Orientación dominante........................... & Procesual & Finalista \\
\hline
\end{tabular}

Fuente: Arias Maldonado, 2004

\section{LAS NECESIDADES DE LAS GENERACIONES FUTURAS: LA EQUIDAD INTER E INTRAGENERACIONAL}

La literatura de referencia en materia de sostenibilidad recoge preguntas sin acuerdo claro en sus respuestas, como son: ¿cuáles son las necesidades en las sociedades contemporáneas? ¿Se trata de necesidades básicas o de necesidades creadas por la sociedad de consumo? ¿Es posible hablar de distintos tipos de necesidades considerando si se trata de sociedades o economías de supervivencia (sin las necesidades básicas cubiertas), o de economías desarrolladas? A su vez: ¿son las necesidades de los seres humanos o de la naturaleza y los ecosistemas que alberga las que importan? ¿y es necesario definir las necesidades no ya desde el presente sino sobre la base de su viabilidad y cambio a medio y largo plazo?

La Unión Mundial de la Conservación en el Programa de Medio Ambiente de las Naciones Unidas y del Fondo Mundial para la Conservación de la Naturaleza (1991) sostienen que el concepto de desarrollo sostenible pone límites al mundo desarrollado, impulsa la mejora de la calidad de vida y fomenta la conciencia sobre los límites de los ecosistemas biofísicos del planeta.

Da así comienzo un nuevo contexto institucional preocupado por la evaluación económica y los conflictos sociales, la distribución de las demandas de la sociedad, la reducción del conflicto social, el papel de las instituciones, las normas y las políticas, los acuerdos políticos 
y sociales que contribuyan al consenso, la accesibilidad equitativa a los recursos naturales y sus beneficios, y la redistribución de los riesgos (O'Connor et al., 1998); más aún, por propuestas que apuestan por un modelo de crecimiento con limitaciones o límites, y que abogue por una ética global inclusivo de las poblaciones del mundo más desfavorecidas (Lafferty et al., 1999: 38).

Ante la interpretación más asentada del crecimiento como algo positivo y evolutivo, Pezzey (1989: xi) en su momento pone de manifiesto que ese tipo de propuestas no suelen consideran la influencia o determinismo del medio ambiente en el bienestar social, mientras que el 'desarrollo' sí lo hace. Añade además que la definición más común del desarrollo sostenible incorpora a las generaciones futuras en ese bienestar social, que el mismo no debe ser inferior al de las actuales, y que la preservación de los recursos no renovables puede resultar más relevante en el análisis de las economías de países en desarrollo, para concluir que muchas definiciones de desarrollo sostenible requieren prestar atención a las necesidades actuales y futuras de las poblaciones pobres.

En la actualidad los modelos de crecimiento predominantes han fracasado en erradicar la pobreza globalmente o en los países, y sigue siendo válido el diagnóstico por el cual "no hay tendencias... u oferta de programas o políticas que ofrezcan alguna esperanza real de disminuir las diferencias existentes entre las naciones ricas y las pobres" (WCED, 1987: xi).

\subsection{La cuestión de la equidad y el desarrollo sostenible}

Si bien la equidad representa un elemento en gran medida determinante para la articulación y seguimiento de la sostenibilidad y el bienestar social, su conceptualización atendiendo a sus vínculos con el desarrollo sostenible no ha sido de manera extensa objeto de reflexión en la literatura, ni en particular por ejemplo desde disciplinas como la economía ecológica (Stymne et al., 2000: 219), que no profundiza mucho más en el ideal que deberíamos de dejar a las generaciones futuras la misma cantidad y variedad de capital.

En el Informe Brundtland se articula a partir de los principios normativos de bienestar y justicia social, de la satisfacción de las necesidades humanas y las aspiraciones sociales (WCED, 1987:43), y en la definición que recoge de desarrollo sostenible - contextualizado en la noción más amplia de necesidad - lo hace aludiendo a dos tipos diferenciados de equidad, como son la equidad intergeneracional y la intrageneracional. La equidad intergeneracional hace referencia a la ecuanimidad en la asignación de recursos de las generaciones actuales hacia las futuras. En este sentido, "la idea central de la sostenibilidad es que las generaciones actuales deben mantener o mejorar las condiciones futuras de vida. Ello implica igualmente que, por ejemplo, "nuestro sistema económico debería de contemplar que las generaciones que nos sigan vivan de nuestros dividendos, manteniendo y mejorando la base de activos para que puedan vivir igual o mejor" (Repetto, 1985: 10).

A su vez, la equidad intrageneracional presta atención al acceso a los recursos entre intereses contrapuestos de las generaciones actuales, con la equidad y el bienestar social como principios, y en especial de los grupos sociales más desfavorecidos.

La equidad, y específicamente la equidad distributiva antes que la eficiencia, la equidad colectiva entre generaciones, o la capacidad para compartir el bienestar entre las generaciones actuales y las poblaciones futuras, son asuntos que van así a irrumpir de manera promitente en el debate que tiene lugar en torno al desarrollo sostenible (Solow, 1974, 1991; Amundsen et al., 1991; Hanley et al., 2001). No en vano, los conceptos de equidad y justicia -en el contexto de rápido deterioro medioambiental- toman forma a 
partir de nociones más amplias, como las de justicia ambiental, distribución social ecológica o distribución intergeneracional del deterioro medioambiental.

En resumen, como parte central de un horizonte prefigurado en el que prime de la solidaridad entre generaciones, se ponen en valor los "derechos" de las generaciones futuras a heredar de las generaciones actuales un entorno natural saludable, contar con los mismos o mejores niveles de calidad ambiental o de aire limpio, no limitando sus posibilidades de existencia o su calidad de vida.

Solow (1991: 3) se muestra más rotundo al afirmar que considerar la equidad o la justicia como objetivos estructuradores de las sociedades contemporáneas implica necesariamente "una obligación, de manera que dejamos a las generaciones futuras la opción o la capacidad de estar tan bien como lo estamos nosotros, aunque no está claro que uno pueda ser más preciso que eso, y donde la sostenibilidad es un mandato judicial no para satisfacernos a nosotros mediante el empobrecimiento de nuestros sucesores".

Finalmente, el conjunto de premisas anteriormente expuestas y que explican la notición institucional de la equidad y el debate en torno a la misma cabe destacar apelan a su vez a la 'futurabilidad' (futurity) -o futuro- (Selman, 2000: 40) y 'universalismo' como estrechamente relacionados (Anand et al., 1994).

'Futurabilidad' dada la visión a largo plazo que se presenta como ineludible al reflexionar sobre la sostenibilidad y el desarrollo sostenible, la justicia y la equidad en sus múltiples posibilidades -la equidad intergeneracional y la justicia social intrageneracional-, la responsabilidad transfronteriza y la equidad geográfica, la equidad procedimental de cara a las personas tratadas de manera abierta y justa, la equidad inter-especies y la importancia de la biodiversidad (Haughton, 1999; Hopwood et al., 2005: 40).

Por su parte, y en estrecha relación con lo anterior, el universalismo atiende a la concienciación en torno al estrecho vínculo entre las necesidades del presente y las del futuro, a la importancia de no dejar de prestar atención a las demandas de los actualmente más desfavorecidos mientras se anticipan las necesidades venideras (Anand et al., 1994).

\section{CONCLUSIONES}

La publicación por Naciones Unidas del Informe Brundtland (WCED, 1987) representa un hito con el que se inicia el debate y reflexión sin precedentes a escala internacional para abordar el reto de la sostenibilidad y el desarrollo sostenible al que se enfrentan la sociedades contemporáneas y que dura hasta la actualidad.

La definición que desde entonces ofrece Naciones Unidad de esta noción ha sido objeto de una intensa discusión entre sus defensores y detractores para ante todo tratar de determinar su alcance semántico y operatividad, y así su capacidad de para transformar el modelo de desarrollo basado en la economía de mercado y la explotación intensa de los recursos naturales el planeta.

En el presente artículo hemos ofrecido una revisión de las posiciones y argumentos que a nuestro juicio consideramos ante todo han articulado el debate institucional y científico en torno a la noción de sostenibilidad y desarrollo sostenible presentada en el Informe Brundtland. La intención es a su vez arrojar luz sobre el concepto.

En dicha exploración, cabe distinguir dos espacios de especialmente significativos, como son el de la sostenibilidad planetaria y las diferencias socioeconómicas entre distintas regiones del mundo; y ante todo, atendiendo al concepto como un término 
"falso", que bajo su terminología "esconde" mantener el estado actual de un modelo de desarrollo insostenible.

Si bien nos encontramos ante un concepto de contrastada influencia intelectual e institucional, el desarrollo sostenible carece de poder real de cambio del modelo hegemónico de desarrollo, causante del impacto ambiental en el planeta, a no ser que realmente se aborde e implemente desde un enfoque 'fuerte' de sostenibilidad.

El primer paso sería reconocer la necesidad de superar el caos de definiciones. Más aún, hoy en día, la literatura sobre el desarrollo sostenible se ha estancado como consecuencia de ese debate. Se necesitan generar procesos nuevos, vías alternativas para la reflexión, que permitan afrontar la realidad social, económica, política, ambiental y cultural desde un punto de vista común acorde al bagaje histórico-cultural de las diferentes sociedades.

En consecuencia, quedan por resolver cuestiones centrales como: ¿se puede decir que los conceptos de sostenibilidad o de desarrollo sostenible realmente representan tanto en su definición y argumentación como en su traducción empírica un cambio sustancial en los principios sobre los que se organiza el modelo actual de desarrollo económico y social?, ¿son superables las contradicciones en las que incurren estos términos?, ¿es la sostenibilidad una quimera?

\section{BIBLIOGRAFÍA}

AMUNDSEN, E. S. y ASHEIM, G. (1991). "The Notion of Sustainable Development”, en The Nordic Journal of Environmental Economics, 2:10-14.

ANAND, S. y SEN, A. (1994). "Sustainable Human Development: Concepts and Priorities", en Office of Development Studies Discussion, Paper $n^{\circ} 1$. Nueva York: UNDP.

ARIAS MALDONADO, M. (2004). "Sustentabilidad y democracia. Hacia una articulación democrática del principio de sustentabilidad”. Revista Española de Ciencia Política, 11:121-148.

BAETEN, G. (2000). "The tragedy of the highway: Empowerment, disempowerment and the politics of sustainability discourses and practices”, en European Planning Studies, 8(1):69-86.

BANCO MUNDIAL (1987). Environment, Growth, and Development. Washington D.C.: World Bank, Development Committee, Pamphlet 14.

BANURI, T. (1999). "Sustainable Development and Climate Change", en Policy Matters nº4.

BEBBINGTON, J. (2001), "Sustainable development: a review of the international development, business and accounting literature", en Accounting Forum, 25(2):128-157.

BERKHOUT, F.; LEACH, M. y SCOONES, I. (eds.) (2003). Negotiating Environmental Change: New Perspectives from Social Science. Londres: Edgar Elgar.

BURGESS, J.; HARRISON, C. M. y FILIUS, P. (1998). "Environmental communication and the cultural politics of environmental citizenship", en Environment and Planning, 30:1445-1460.

CAMPBELL, S. (1996). "Green Cities, Growing Cities, Just Cities? Urban Planning and the Contradictions of Sustainable Development". Journal of the American Planning Association, 62(3): 296-312.

CARVALHO, G.O. (2001). "Sustainable development: is it achievable within the existing international political economy context?". Sustainable Development, 9(2):61-73.

CENDRA GARRETA, J. y STAHEL, A. W. (2006). "Hacia una construcción social del desarrollo sostenible basada en la definición de sus dimensiones y principios, articulados a partir de la ecuación IPAT. Aproximación a sus implicaciones y debates". Revista Internacional Sostenibilidad, Tecnología y Humanismo, 1:1-32.

CENTRE DE COOPERATION INTERNATIONALE EN RECHERCHE AGRONOMIQUE POUR LE DEVELOPPEMENT - CIRED (1986). "The introduction of upland rice in the cropping systems in the 'Hauts de l'Ouest' area”. Fiche d'Essai, n' 16. 
CHRISTIE, I. y WARBURTON, D. (2001). From Here to Sustainability: Politics in the Real World. London: Earthscan.

COHEN, C. (1971). Democracy. Nueva York: The Free Press.

COMMISSION OF THE EUROPEAN COMMUNITIES - CEC (1992). Towards Sustainability. A European Union Programme of Policy and Action in Relation to the Environment and Sustainable Development, COM 92(23). Bruselas.

DALAL-CLAYTON, B. y BASS, S. (2004). What is sustainable development?, accesible en http:// issuu.com/sustainabledevelopers/docs/what_is_sustainable_development [visitado 6/5/2015].

DALE, A. (2001). At the Edge. Sustainable Development in the 21st Century. Vancouver: University of British Columbia Press.

DALY, H. E. (1996). Beyond Growth. Boston: Beacon Press.

DAVIDSON, J. (2000). "Sustainable development: business as usual or a new way of living?", en Environmental Ethics, 22(1):25-42.

DAWE, N.K. y RYAN, K.L. (2003). "The faulty three-legged stool model of sustainable development", en Conservation Biology, 17(5):1458-1460.

DOBSON, A. (1999). Justice and the Environment. Conceptions of Environmental Sustainability and Theories of Distributive Justice. Oxford: Oxford University Press.

DOBSON, A. (1998). Justice and the Environment. Conceptions of Environmental Sustainability and Dimensions of Social Justice, Oxford: Oxford University Press.

DRESNER, S. (2002). The Principles of Sustainable Development. London: Earthscan Publications.

EDWARDS-JONES, G.; DAVIES, B. y HUSSAIN, S. (2000). Ecological Economics: An Introduction. Oxford: Blackwell Science.

EKINS, P. (1993). "Limits to growth and Sustainable Development. Grappling with Ecological Realities", en Ecological Economics, 8:269-288.

EKINS, P. y JACOB, M. (1998). "Ecological Distribution and Distributed Sustainability", en S. Faucheux, M. O'Connor y J. Van der Straaten (eds.) Sustainable Development: Concepts, Rationalities and Strategies. Dordecht: Kluwer academic publishers, pp.33-56.

FABER, M.; PETERSEN, T. y SCHILLER, J. (2002). "Homo oeconomicus and homo politicus in Ecological Economics" en Ecological Economics, 40(3):323-333.

FAUCHEUX, S.; O'CONNOR, M. y VAN DER STRAATEN, J. (eds.) (1998). Sustainable Development: Concepts, Rationalities and Strategies. Londres: Kluwer academic publishers.

FERNANDO, J. (2003). "The power of unsustainable development: what is to be done?" en Annals of the American Association of Political and Social Sciences, vol. 590:6-34.

FOLADORI, G. (1999). "Sustentabilidad ambiental y contradicciones sociales". Ambiente \& Sociedade, 5: 19-34.

FONT, N. y SUBIRATS, J. (2000). Local y Sostenible. La Agenda 21 Local en España. Barcelona: Icaria.

FRAZIER, J. (1997). "Sustainable development: modern elixir or sack dress?" en Environmental Conservation, 24(2):182-193.

GARCÍA, E, (2004). Medio ambiente y sociedad: La civilización industrial y los límites del planeta. Madrid: Alianza Editorial.

GIBBS, D. C. (1998). "Regional development agencies and sustainable development", en Regional Studies, 32(4):365-8.

GIDDINGS, B.; HOPWOOD, B. y O'BRIEN, G. (2002). "Environment, economy and society: fitting them together into sustainable development", en Sustainable Development, 10(4):187-196.

GODARD, O. (1998). "Sustainable Development and the process of justifying choices in a controversial universe", en S. Faucheux; M. O'Connor y J. Van der Straaten (eds.) Sustainable Development: Concepts, Rationalities and Strategies, pp. 299-316.

GOLDIN, I. y WINTERS, L.A. (1995). The Economics of Sustainable Development. Cambridge: University of Cambridge Press.

GOW, D. (1992). "Poverty and Natural Resources: Principles for Environmental Management and Sustainable Development". Environmental Impact Assessment Review, 12(1-2): 49-65. 
HANLEY, N.; SHOGREN, J.F. y WHITE, B. (2001). Introduction to Environmental Economics. Oxford: Oxford University Press.

HARRISON, N.E. (2000). Constructing Sustainable Development. Nueva York: State University of New York Press.

HAUGHTON G. (1999). "Environmental justice and the sustainable city", en D. Satterthwaite (ed.) The Earthscan Reader in Sustainable Cities. London: Earthscan, pp-62-79.

HOLMBERG, J.; ROBÈRT, K.H. y ERIKSSON, K.E. (1994). "Socio-Ecological Principles for a Sustainable Society", en R. Costanza; S. Olman y J. Martinez-Alier (eds.) Getting Down to Earth Practical Applications of Ecological Economics. Washington D.C.: Island Press.

HOPWOOD, B.; MELLOR, M. y O'BRIEN, G. (2005). "Sustainable development: mapping different approaches", en Sustainable Development, 13(1):38-52.

HOWARTH, R.B. y WILSON, M.A., (2006). "A theoretical approach to deliberative valuation: aggregation by mutual consent”, en Land Economics, 82(1):1-16.

HUETING, R. (1986). “An economic scenario for a conserver economy”, en P. Ekins (ed.), The living Economy: a new economics in the making. Londres: Routledge and Kegan Paul, 244-256.

INTERNATIONAL UNION FOR CONSERVATION OF NATURE AND NATURAL RESOURCES (IUCN) (1980). World Conservation Strategy: Living Resource Conservation for Sustainable Development), en colaboración con la United Nations Environment Programme (UNEP) y la World Wildlife Fund (WWF). Gland, Switzerland: IUCN World Headquarters.

IUCN/UNEP/WWF (1991). Caring for the Earth: A Strategy for Sustainable Living. Gland, Switzerland.

JABAREEN, Y. (2008). "A new conceptual framework for sustainable development", en Environmental Development and Sustainability, 10(2):179-192.

JACOBS, M. (1991). The Green Economy. Londres: Pluto Press.

JORDAN, A. y ADGER, N. (eds.) (2009). Goberning Sustainability. Cambridge: Cambridge University Press.

KABEER, N. (2005). "The search for inclusive citizenship: Meanings and expressions in an interconnected world", en J. Gaventa Claiming citizenship: rights, participation and accountability. Londres: Zed Books.

KANE, M. (1999). "Sustainability concepts: From theory to practice", en Köhn, J. et al. (eds.), Sustainability in question. The search for a conceptual framework. Cheltenham: Edward Elgar.

KENNY, M. y MEADOWCROFT, J. (1999). Planning sustainability. Londres: Routledge.

LAFFERTY, W. M. y LANGHELLE, O. (eds.) (1999). Towards sustainable development: on the goals of development - and the conditions of sustainability. Houndmills: MacMillan Press Ltd.

LAFFERTY, W.M. (2004). Governance for Sustainable Development. The Challenge of Adapting Form to Function. Cheltenham: Elgar.

LAFFERTY, W.M. y MEADOWCROFT, J. (eds.) (2000). “Concluding perspectives”, en W. Lafferty y J. Meadowcroft (eds.) Implementing Sustainable Development: Strategies and Initiatives in High Consumption Societies. Oxford: Oxford University Press.

LAMBERTON, G. (2005), Sustainable sufficiency-an internally consistent version of sustainability, Sustainable Development, vol. 13(1):53-78.

LÉLÉ, S. (1991). "Sustainable development: a critical review" en World Development, 19 (6): 607-621. LUFFIEGO GARCÍA, M. y RABADÁN VERGARA, J.M. (2000). "La evolución del concepto de sostenibilidad y su introducción en la enseñanza”, en Enseñanza de las Ciencias, 18(3):473-486.

LUKE, T. (1995). "Sustainable development as a power/knowledge system: the problem of govern mentality", en F. Fischer y M. Black (eds.) Greening Environmental Policy: the Politics of a Sustainable Future. Nueva York: St. Martin's Press, pp. 21-32.

MEBRATU, D. (1998). "Sustainability and Sustainable Development: historical and conceptual review", en Environmental Impact Assessment Review, 18(6):493-520.

MEBRATU, D. (1996). Sustainability as a Scientific Paradigm. Lund: International Institute for Industrial Environmental Economics.

MIDDLETON, N.; O'KEEFE, P. y MOYO, S. (1993). The Tears of the Crocodile: from Rio to Reality in the Developing World. London: Pluto. 
MITCHAM, C. (1995). "The Concept of Sustainable Development: its Origins and Ambivalence". Technology In Somy, 17(3): 311-326.

MITLIN, D. (1992). "Sustainable Development: A Guide to the Literature", en Environment and Urbanization, 4(1):111-124.

MOZAFFAR, Q. (2001). "Sustainable development: Concepts and rankings". Journal of Development Studies, 3: 134-161.

MYERSON, G. y RYDIN, Y. (1996). The language of the environment. A new rhetoric. Londres: UCL Press.

NACIONES UNIDAS (1992). Agenda 21. United Nations Conference on Environment and Development (UNCED). Conches, Switzerland.

NAREDO, J. M. (1996). Sobre el Origen, el Uso y el Contenido del Término Sostenible, accesible en http://habitat.aq.upm.es/cs/p2/a004.html [visitado 24/9/2014].

NEWMAN, L. (2006). "Change, uncertainty, and futures of sustainable Development", en Futures, 38:633-637.

O’CONNOR, J. (2002). “Es posible el capitalismo sostenible?”, en H. Alimonda (comp.) Ecología política. Naturaleza, sociedad y utopía. Buenos Aires: CLACSO, pp. 27-52.

O'CONNOR, M. (ed.) (1994). Is Capitalism Sustainable? Political Economy and Politics of Ecology. Nueva York: Guilford Publications.

O'CONNOR, M. y MARTÍNEZ ALIER, J. (1998). "Ecological Distribution and Distributed Sustainability”, en: S. Faucheux; M. O'Connor y J. Van der Straaten (ed.) Sustainable Development: Concepts, Rationalities and Strategies. London: Kluwer Academic Publishers, pp. 33-53.

O'RIORDAN, T. (1993). Interpreting the Precautionary Principle. CSERGE Working Paper PA 9303. UK: CSERGE.

PAEHLKE, R. (1995). "Environmental values for a sustainable society: the democratic challenge", en F. Fischer y M. Black (eds.) Greening Environmental Policy. Londres: Paul Chapman Publishing Ltd, pp. 129-144.

PEZZEY, J. (1989). "Economic Analysis of Sustainable Growth and Sustainable Development", en World Bank Environment Department, Working Paper $\mathrm{n}^{\circ} 15$. Washington D.C.

PEZZOLI, K. (1997). "Sustainable development: a transdisciplinary overview of the literature". Journal of Environmental Planning and Management, 40(5): 549-574.

Programa de las Naciones Unidas para el Medio Ambiente - PNUMA - (1973), Naciones Unidas.

REDCLIFT, M. R. (1993). "Sustainable development: Concepts, contradictions, and conflicts", en P. Allen (ed.), Food for the future: Conditions and contradictions of sustainability. Nueva York: John Wiley.

REDCLIFT, M. R. (1987). Sustainable development: Exploring the contradictions. Nueva York: John Wiley and Sons.

REDCLIFT, M. R. y WOODGATE, G. (1997). Sociología del Medio Ambiente. Una perspectiva internacional. Madrid: Mc. Graw Hill.

REPETTO, R. (ed.) (1985). The global possible: Resources, development, and the new century. New Haven, CT: Yale University Press.

RICHARDSON, D. (1997). "The Politics of Sustainable Development". En: S. Barker et al. (eds.) The Politics of Sustainable Development: Theory and Practice within the European Union. London: Routledge.

RIGGS, F. W. (1984). "Development”, en G. Satori (ed.) Social Science Concepts: A Systematic Analysis. Beverly Hills, CA: Sage Publications.

ROBINSON, J. (2004). "Squaring the circle? Some thoughts on the idea of sustainable development", en Ecological Economics, 48(4):369-384.

ROSENAU, J. (2003). Distant Proximities: Dynamics beyond Globalization. Princeton, NJ: Princeton University Press.

SACHS, I. (1994). "Environnement, développement, marché", en Natures Sciences Sociétés, 2(3):258265.

SACHS, I. (1991). "Le Sud et la Conférence de Río de Janeiro", en AA.VV. Environnement et gestion de la planète. París: Cahiers Francais, La Documentation Francaise. 
SACHS, W. (2002). The Jo'burg Memo: Fairness in a Fragile World. Memorandum for the World Summit on Sustainable Development. Berlin: Heinrich Böll Foundation - World Summit papers.

SACHS, W. (1999). "Sustainable development and the crisis of nature: on the political anatomy of an oxymoron", en F. Fischer y M. Hajer (eds.) Living with Nature: Environmental Politics as Cultural Discourse. Oxford: Oxford University Press.

SATTERTHWAITE, D. (1996). "For better living", en Down to Earth, 5(5):31-35.

SCOONES, I., et al. (2007). Dynamic Systems and the Challenge of Sustainability. Brighton: Steps working paper 1 .

SELMAN, P. (2000). “A Sideways Look at Local Agenda 21”, en Journal of Environmental Policy \& Planning, 1(1):39-53.

SIVARAKSA, S. (1992). "The religion of consumerism", en S. Sivaraksa Seeds of Peace: A Buddhist Vision for Renewing Society. Berkeley, CA.: Parallax Press, pp. 3-10.

SNEDDON, C. (2000). "Sustainability in ecological economics, ecology and livelihoods: a review", en Progress in Human Geography, 24(4):521-549.

SNEDDON, C.; HOWARTH, R. B. y NORGAARD, R. B. (2006). "Sustainable development in a postBrundtland world". Ecological Economics, 57(2): 253-268.

SOLOW, R. (1991). "Sustainability: An Economist's Perspective", en R. Dorfman y N.S. Dorfman (eds.) Economics of the Environment. Nueva York: WW Norton \& Company.

SOLOW, R. (1974). "Intergenerational Equity and Exhaustible Resources". Review of Economic Studies, 41: 29-45.

STOW, D.A.V. (1989). "Geological aid to developing countries", en Journal of the Geological Society, 146(1):187-189.

STRAATEN, J. (ed.) (1989). Sustainable Development: Concepts, Rationalities and Strategies. Dordecht: Kluwer academic publishers.

STYMNE, S. y JACKSON, T. (2000). Intra-generational equity and sustainable welfare: A time series analysis for the UK and Sweden. Ecological Economics, 33, 219-236.

TRYZNA, T.C. (1995). A Sustainable World. Sacramento: IUCN.

VICTOR, P. (1991). "Indicators of sustainable development: some lessons from capital theory", en Ecological Economics, 4(3):191-213.

VILLANUEVA, C. (1997). "Community development and the futures of sustainable communities in the Philippines", en Y. Kaoru (ed.) Sustainable global community in the information age: Vision from future studies. Praeger, CT.: Praeger Studies on the 21 st Century.

VOLGER, J. y JORDAN, A. (2003). "Governance and the environment”, en F. Berkhout; M. Leach y I. Scoones (eds.) Negotiating Environmental Change: New Perspectives from Social Science. Londres: Edgar Elgar.

WALLNER, H.; NARODOSLAWSKY, M. y MOSER, F. (1996). "Islands of sustainability: a bottomup approach towards sustainable development”, en Environment and Planning, 28(10):1763-1778.

WETLESEN, J. (1993). "Who has a moral status in the environment?" en Trumpeter, 23(1):3-27.

WORLD COMMISSION ON ENVIRONMENT AND DEVELOPMENT (WCED) (1987). Our common future. The Brundtland Report. Oxford: Oxford University Press.

WORSTER, D. (1995). “The shaky ground of sustainability”, en G. Sessions (ed.) Deep Ecology for the Twenty-First Century. Boston, MA: Shambhala, pp. 417-427. 


\section{Breve currículo:}

\section{Iván López Pardo}

Doctor en Análisis y Evaluación de Procesos Políticos y Sociales por la Universidad Carlos III de Madrid, profesor en la Facultad de Ciencias Sociales y del Trabajo en el Departamento de Psicología y Sociología de la Universidad de Zaragoza. Especialidades: sociología medioambiental, la sostenibilidad y desarrollo sostenible y, más recientemente, en el análisis de cambios socio-políticos en España. 